\title{
Exercise therapy for spondyloarthritis: a systematic review
}

\begin{tabular}{|l|l|}
\hline Item type & Systematic Review \\
\hline Authors & O'Dwyer, Tom; O'Shea, Finbar; Wilson, Fiona \\
\hline Citation & $\begin{array}{l}\text { Exercise therapy for spondyloarthritis: a systematic review } \\
\text { 2014 Rheumatology International }\end{array}$ \\
\hline DOI & 10.1007/s00296-014-2965-7 \\
\hline Publisher & Rheumatology international \\
\hline Journal & Rheumatology International \\
\hline Rights & Archived with thanks to Rheumatology International \\
\hline Downloaded & 21-Jun-2016 12:17:32 \\
\hline Link to item & http://hdl.handle.net/10147/317552 \\
\hline
\end{tabular}




\title{
Exercise therapy for spondyloarthritis: a systematic review
}

\author{
Tom O'Dwyer · Finbar O'Shea · Fiona Wilson
}

Received: 23 December 2013 / Accepted: 4 February 2014

(C) Springer-Verlag Berlin Heidelberg 2014

\begin{abstract}
To evaluate the effects of therapeutic exercise on pain, stiffness, quality of life, physical function, disease activity, health-related fitness and cardiovascular risk factors in adults with spondyloarthritis (SpA). Electronic databases (Cochrane Central Register of Controlled Trials, EMBASE, MEDLINE/PubMed, PEDro, AMED, CINAHL) were systematically searched from inception to October 2013 using medical subject headings and keywords. This was supplemented by searching conference abstracts and a hand search of reference lists of included studies. Randomised and quasi-randomised studies of adults with SpA in which at least one of the comparison groups received an exercise intervention were included. Outcomes of interest were pain, stiffness, quality of life, physical function and disease activity. Secondary outcomes were health-related fitness and cardiovascular risk factors. Two reviewers independently screened studies for inclusion. Methodological quality was assessed by two reviewers using the Cochrane risk of bias tool and the PEDro scale. Twenty-four studies, involving 1,498 participants, were included. Meta-analyses were not undertaken due to clinical heterogeneity, and this review focuses on qualitative synthesis. Moderate evidence supports exercise interventions in improving physical
\end{abstract}

Electronic supplementary material The online version of this article (doi:10.1007/s00296-014-2965-7) contains supplementary material, which is available to authorized users.

T. O’Dwyer $(\bowtie) \cdot$ F. Wilson

Discipline of Physiotherapy, School of Medicine, Trinity Centre

for Health Sciences, Trinity College, Dublin, Ireland

e-mail: odwyertk@tcd.ie

F. O'Shea

Department of Rheumatology, St. James's Hospital,

Dublin 8, Ireland function, disease activity and chest expansion compared to controls; there is low-level evidence of improved pain, stiffness, spinal mobility and cardiorespiratory function. Supervised group exercise yields better outcomes than unsupervised home exercise. The addition of aerobic components to flexibility programmes improves cardiorespiratory outcomes, but not cardiovascular risk factors. The most effective exercise protocol remains unclear. Current evidence suggests that therapeutic exercises are beneficial for adults with ankylosing spondylitis; effects on other SpA subtypes are unknown.

Keywords Ankylosing spondylitis · Spondyloarthritis · Exercise $\cdot$ Fitness $\cdot$ Cardiovascular risk

\section{Introduction}

The spondyloarthropathies (SpA) are a heterogeneous group of inflammatory arthritides that include ankylosing spondylitis (AS), reactive arthritis (ReA), enteropathic spondylitis or arthritis associated with irritable bowel disease (IBD), psoriatic arthritis (PsA) and undifferentiated spondyloarthropathy (uSpA) [1]. They are characterised by sacroiliitis with inflammatory back pain, peripheral joint pain, enthesitis, dactylitis and extra-articular manifestations including uveitis, psoriasis and IBD. SpA are associated with decreased physical function and lower health-related quality of life (QoL) [2,3]. Depending on clinical features and imaging, SpA can be classified as predominantly axial $\mathrm{SpA}$ or predominantly peripheral SpA $[4,5]$.

Current practice guidelines recommend a combination of pharmacological and non-pharmacological treatment modalities for optimal management of patients with AS and PsA [6, 7]. Exercise programmes have shown small 
but beneficial effects on spinal mobility and physical function in adults with AS [8]. To our knowledge, no review has systematically examined the effect of exercise in other SpA subtypes, although exercise is frequently advised as part of their management.

Epidemiological evidence suggests that AS and PsA are associated with elevated cardiovascular risk factors and increased cardiovascular morbidity and mortality [9, 10]; however, to date, studies have not explored the effects of therapeutic exercise on cardiovascular risk factors and physical fitness parameters in SpA. The aims of this review were to assess the effects of exercise on pain, stiffness, QoL, physical function, health-related fitness and cardiovascular risk factors in adults with $\mathrm{SpA}$.

\section{Materials and methods}

A protocol outlining the review strategy and methods of analysis was registered with a registry of systematic reviews (available at http://www.crd.york.ac.uk/PROSPERO/display_record.asp?ID=CRD42013004015).

\section{Eligibility criteria}

Adults diagnosed by a rheumatologist as having AS, ReA, PsA, uSpA or enteropathic spondylitis were included. Participants under 18 years of age or with juvenile-onset SpA were excluded. Quasi-randomised and randomised controlled trials (RCT) in which at least one of the groups received exercise therapy were included. Review articles, observational studies without controls, case reports, crosssectional studies and commentaries were excluded.

For the purpose of this review, exercise-based interventions comprised one or more of the following components: range of motion (stretching), strengthening or aerobic exercise. Any dosages of exercise prescription (i.e. any frequency, intensity, mode or duration) were considered. However, interventions offering general advice to exercise without prescribing specific exercises were excluded. Exercise-based interventions delivered in an inpatient setting were excluded, unless being compared to a distinct outpatient exercise group. Studies in which exercise-based interventions were administered in conjunction with other modalities (e.g. manual therapy) were excluded.

The primary outcomes of interest to this review were pain, stiffness, disease activity, physical function and QoL. Secondary outcome variables were health-related fitness measures (cardiorespiratory, muscular strength, flexibility and body composition) and cardiovascular risk factors (blood pressure, glycaemia, metabolic syndrome, body mass index and lipid profile).
Information sources and study selection

Studies were retrieved by searching electronic databases (MEDLINE/PubMed, EMBASE, PEDro, AMED, CINAHL and The Cochrane Central Register of Controlled Trials) from their inception to October 2013. Search terms were adapted for use with each database. Common keywords and medical subject headings related to three components: (1) the condition (e.g. spondyloarthritis), (2) the intervention (e.g. exercise) and (3) the study design (e.g. clinical trial) (See Supplement 1). No search restrictions (date or language) were imposed. The electronic database search was supplemented by searching abstracts of the annual meetings of the World Confederation for Physical Therapy (2003-2011), the American College of Rheumatology (2006-2012), the European League Against Rheumatism (2002-2013) and the American Physical Therapy Association (2002-2013). When only abstracts were available in the published literature, authors were contacted seeking full texts of relevant studies. Finally, a hand search of the reference lists of included studies was conducted.

Two reviewers (TOD and FW) independently screened titles and abstracts to identify studies that potentially met the eligibility criteria. Full texts of these reports were retrieved and independently assessed for eligibility by the same two reviewers. Any disagreements on inclusion were resolved by discussion to achieve consensus, and failing agreement, a third reviewer (FOS) was consulted.

Data collection and analysis

A data extraction template based on Cochrane guidelines [11] was adapted and piloted on five randomly selected studies and modified accordingly. One reviewer (TOD) recorded (1) participant characteristics, (2) details of interventions and (3) relevant outcome data (group means and standard deviations). For continuous data, the differences in group means (with $95 \% \mathrm{CI}$ ) were calculated at clinically relevant time points (i.e. post-intervention and at follow-up). For continuous data reported on different scales, standardised mean differences with $95 \%$ CIs were used. The calculations employed a random effects model. In trials comparing two similar exercise groups and one control group, the exercise group results were pooled for comparative purposes $[12,13]$. In the event that the published data from included studies were insufficient to calculate pooled effects, study authors were contacted requesting additional data. Meta-analyses were planned but ultimately deemed inappropriate due to the heterogeneity of study designs and interventions. Due to the absence of studies exploring the effects of exercise interventions on predominantly axial and predominantly peripheral $\mathrm{SpA}$, this proposed subgroup analysis was 
not completed. Statistical analysis was conducted using Review Manager 5.2 and SPSS 21.

Risk of bias and levels of evidence

A risk of bias appraisal of included studies was performed independently by two reviewers (TOD and FW). Disagreements between the reviewers were resolved through discussion to achieve consensus. Failing agreement, a third reviewer (FOS) arbitrated. The Cochrane Collaboration's risk of bias tool rated risk of bias across six domains as low, high or unclear [11]. The Physiotherapy Evidence Database (PEDro) scale rated methodological quality from 0 (low) to 10 (high) [14]. Fair-to-good reliability has been established for the total PEDro score [15]. Each study was ascribed a level of evidence according to the criteria of the Oxford Centre for Evidence-based Medicine [16] (Table 1). These levels of evidence provide a hierarchy of the likely best evidence. Quality of evidence for key outcomes across comparisons was evaluated following the GRADE levels of evidence [17] (Table 2).

\section{Results}

Study selection

The electronic database search returned 450 records (after the removal of duplicates), and an additional nine reports

Table 1 Oxford Centre for Evidence-based Medicine 2011 levels of evidence

\begin{tabular}{ll}
\hline Level of evidence & Description \\
\hline Level I & $\begin{array}{c}\text { Systematic review of randomised trials or } n \text {-of-1 } \\
\text { trials } \\
\text { Randomised trial or observational study with } \\
\text { dramatic effect }\end{array}$ \\
Level II & $\begin{array}{l}\text { Non-randomised controlled cohort/follow-up } \\
\text { study }\end{array}$ \\
Level IV & $\begin{array}{c}\text { Case-series, case-control or historically con- } \\
\text { trolled studies }\end{array}$ \\
Level V & Mechanism-based reasoning \\
\hline
\end{tabular}

were identified from the search of conference abstracts. One unpublished full-text report of a conference abstract was provided [18]; attempts to obtain full texts of other published abstracts from the authors were unsuccessful. The search strategy and selection process are summarised in Fig. 1. A total of 24 studies (18 RCT) published between 1993 and 2013 were included in this review.

\section{Study characteristics}

Study characteristics and findings are summarised in Table 3. A total of 1,460 participants with AS and 38 fulfilling the Amor criteria for SpA were included. No other SpA subtypes were examined in the included studies. The mean study sample size was 62 (SD 37; range 20-155). The ratio of participants was approximately 3:1 (male:female). Subject characteristics varied in age, disease duration, disease severity and medication use.

Exercise intervention duration ranged from 3 weeks [19] to 3 years [20]. Frequency of exercise varied from twice daily [19] to once weekly [21-23], with individual session duration ranging from $30 \mathrm{~min}[18,24-29]$ to $3 \mathrm{~h}$ [13]. Traditional therapeutic exercises targeting flexibility, posture and respiration predominated. Other exercise protocols included aerobic exercise, strength training, proprioceptive exercise, the Global Posture Re-education (GPR) method, the Pilates method, hydrotherapy and sporting activities. Seven studies compared the effect of exercise to controls, twelve compared two or more types of exercisebased interventions, and five compared therapeutic exercise to another treatment modality (inpatient rehabilitation, balneotherapy, incentive spirometry or spa-exercise therapy).

Self-report measures of physical function, QoL, disease activity, pain and stiffness were identified as primary outcomes. Flexibility was the most commonly examined health-related fitness component; fewer studies examined cardiorespiratory fitness, while no studies examined body composition or muscular strength. Cardiovascular risk factors (cholesterol and triglycerides) were assessed in one study [29]. Follow-up measures were recorded in five studies [24, 30-33].

Table 2 GRADE levels of evidence

Quality level Definition

High We are confident that the true effect lies close to that of the estimate of the effect

Moderate We are moderately confident in the effect estimate: the true effect is likely to be close to the estimate of the effect, but there is the possibility that it is substantially different

Low Our confidence in the effect estimate is limited: the true effect may be substantially different from the estimate of the effect

Very low We have very little confidence in the effect estimate: the true effect is likely to be substantially different from the estimate of effect 
Fig. 1 PRISMA flow diagram of study selection process

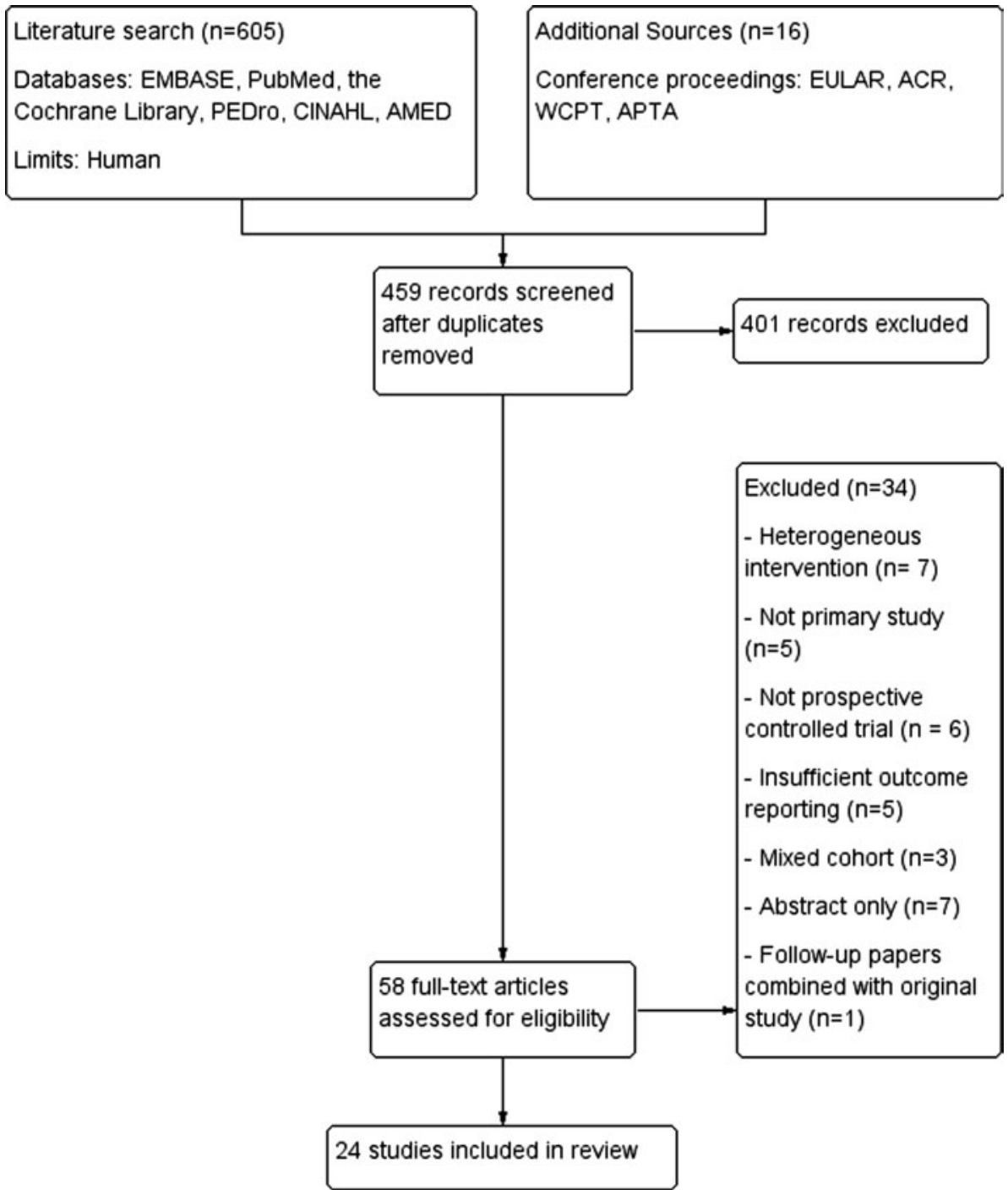

Risk of bias within studies

Synthesis of results

This review focused on a qualitative synthesis of the studies. A meta-analysis was not undertaken due to the heterogeneity of study designs, participants, interventions and reported outcome measures. When sufficient data were reported, individual effect sizes were calculated and presented on forest plots to provide a visual overview of results. Study results are summarised in Table 3.

Comparison 1: therapeutic exercise compared to controls

Seven studies compared therapeutic exercise interventions with controls. Three of these examined home exercise programmes (HEP) [28, 35, 37], two implemented supervised group exercise (GE) [30, 36], and one study conducted Pilates training [34]. One three-armed study compared an unsupervised GPR programme to a HEP and to controls; 


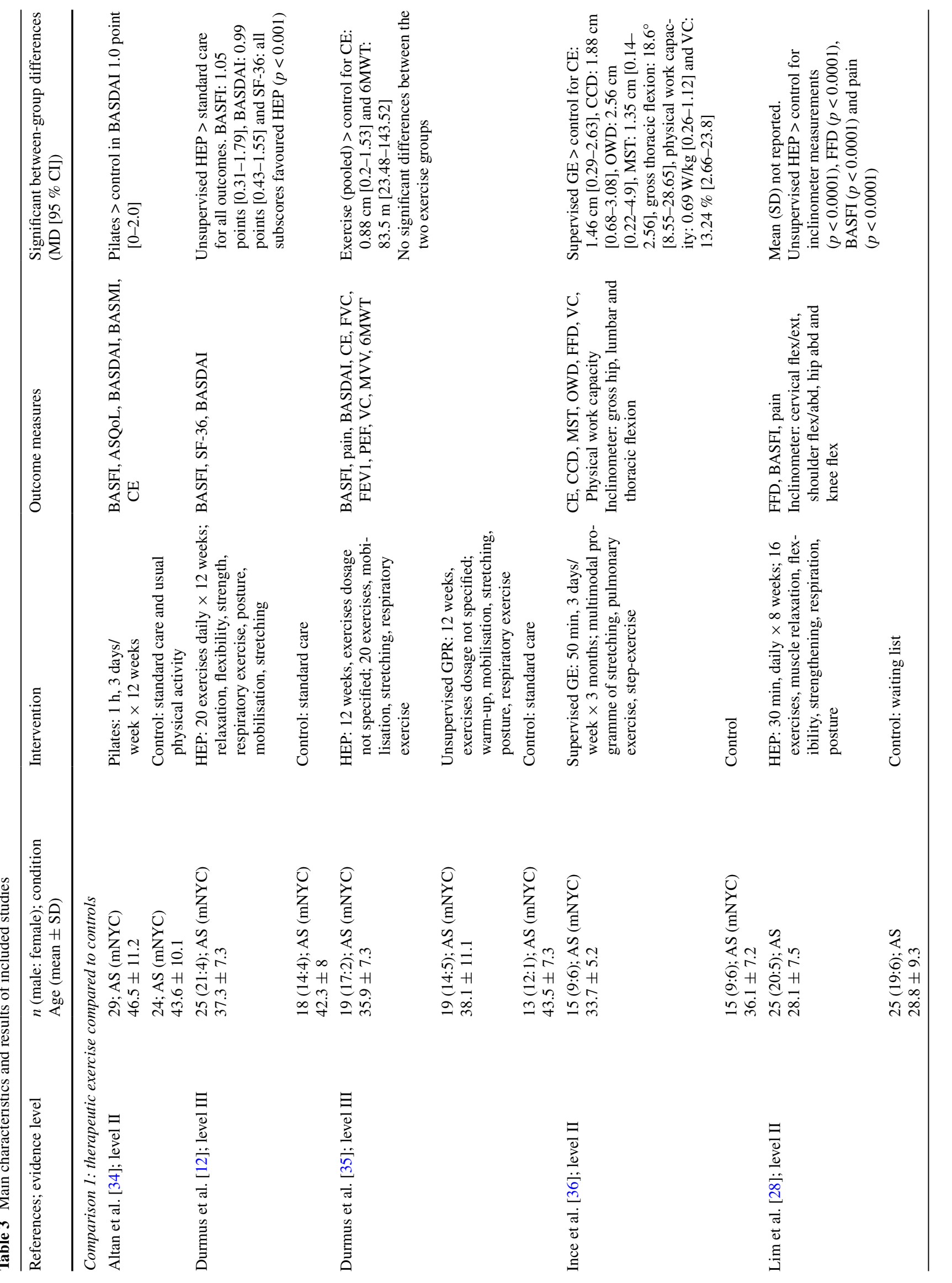




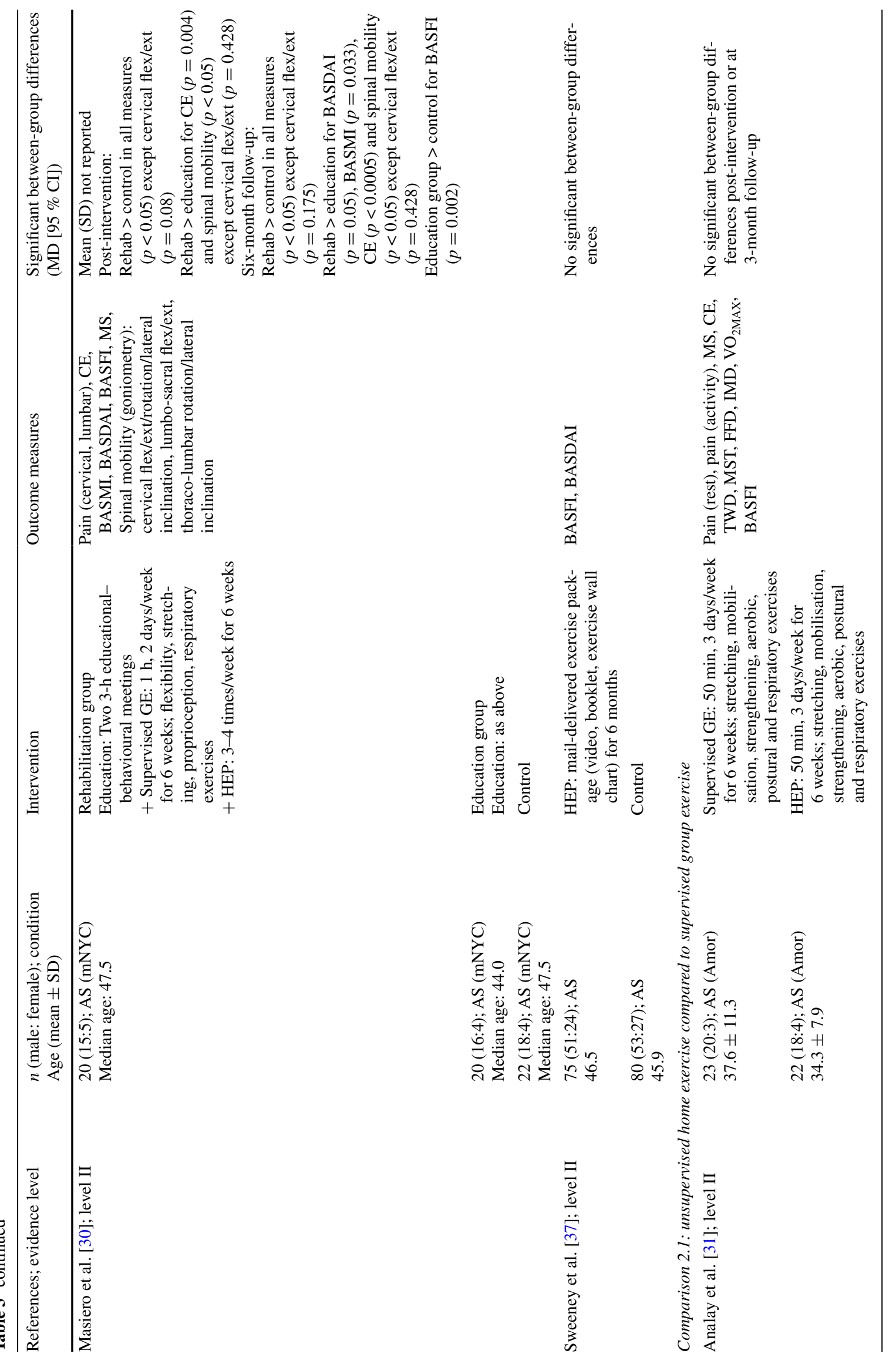




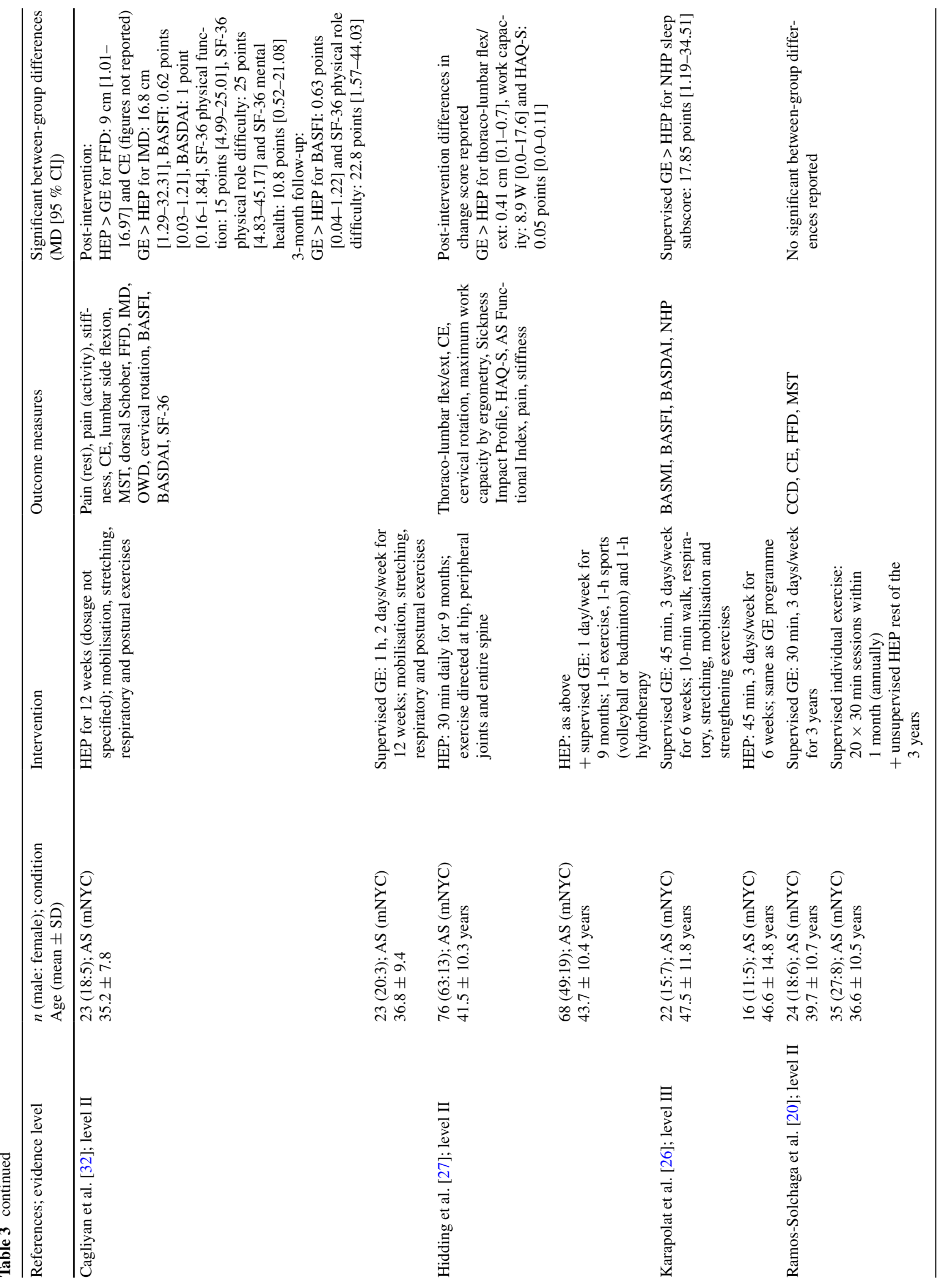




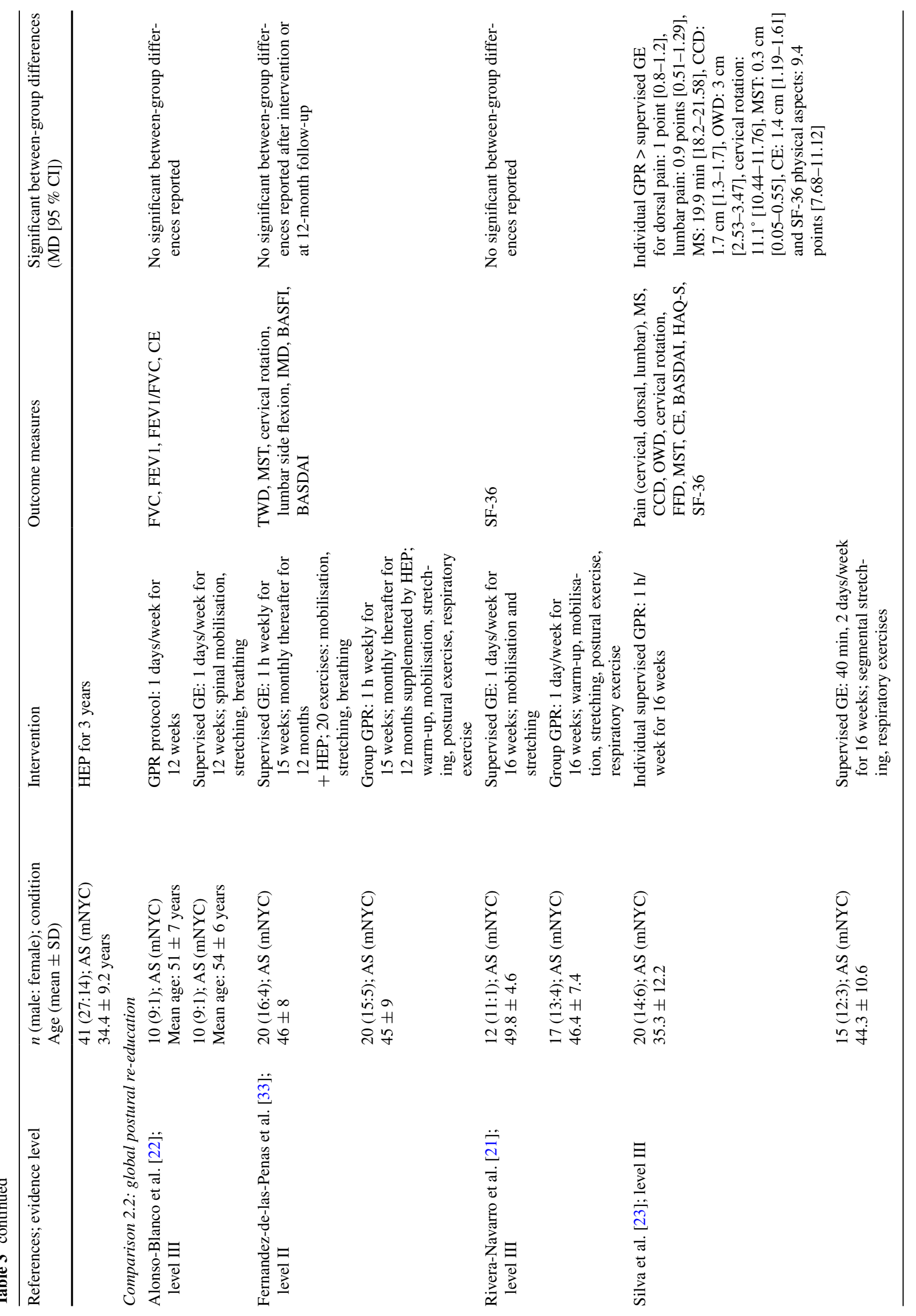




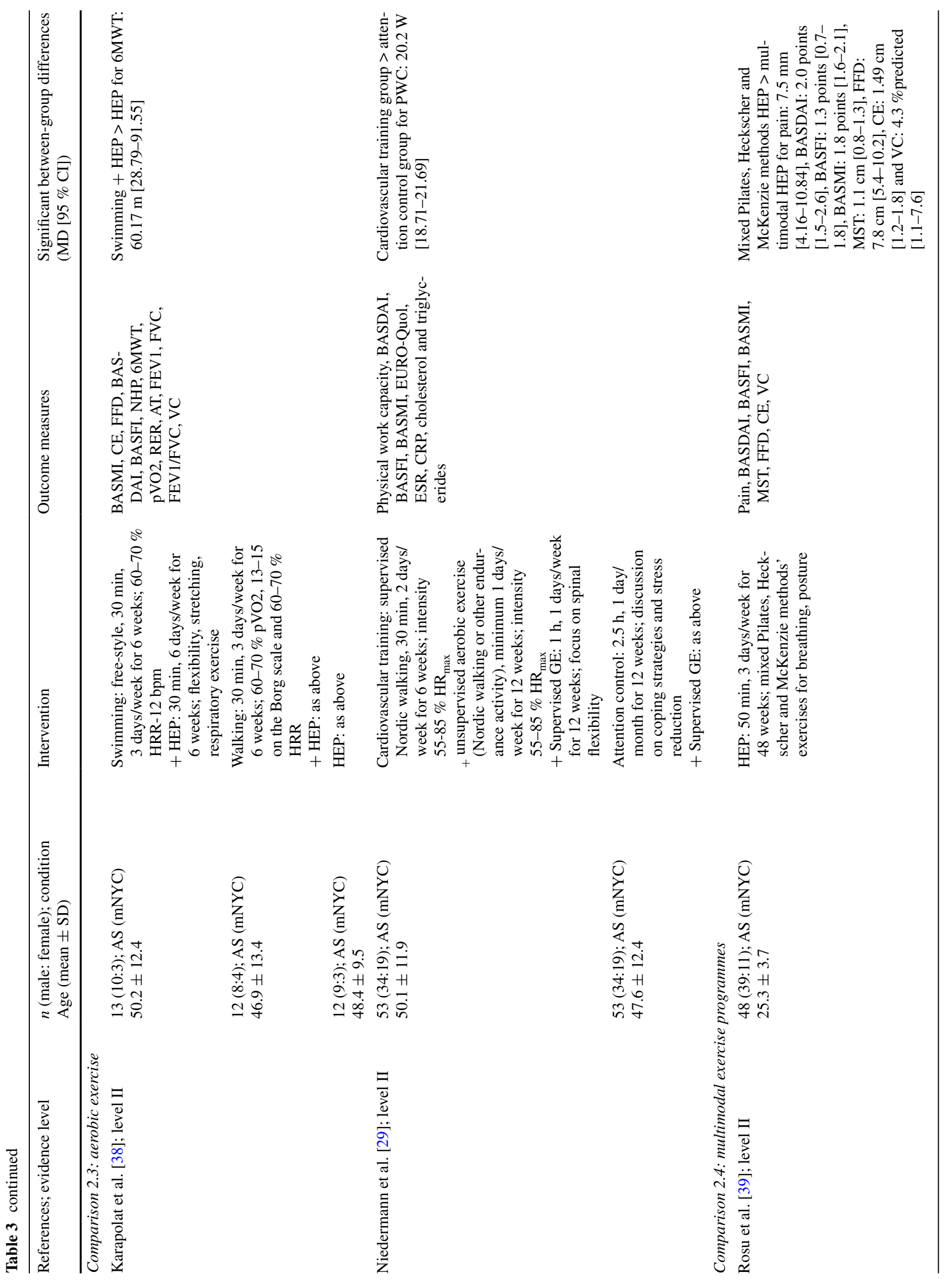




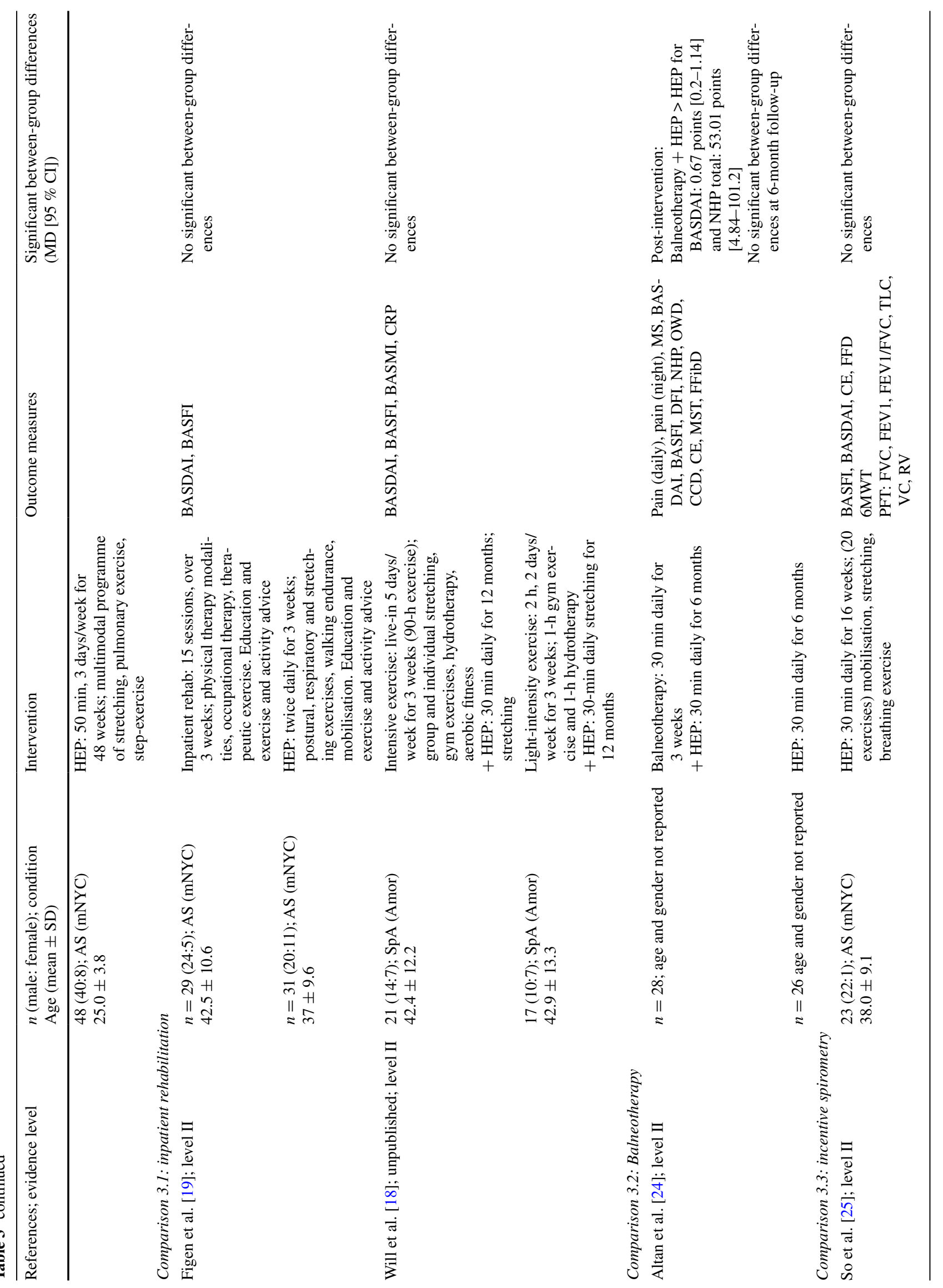




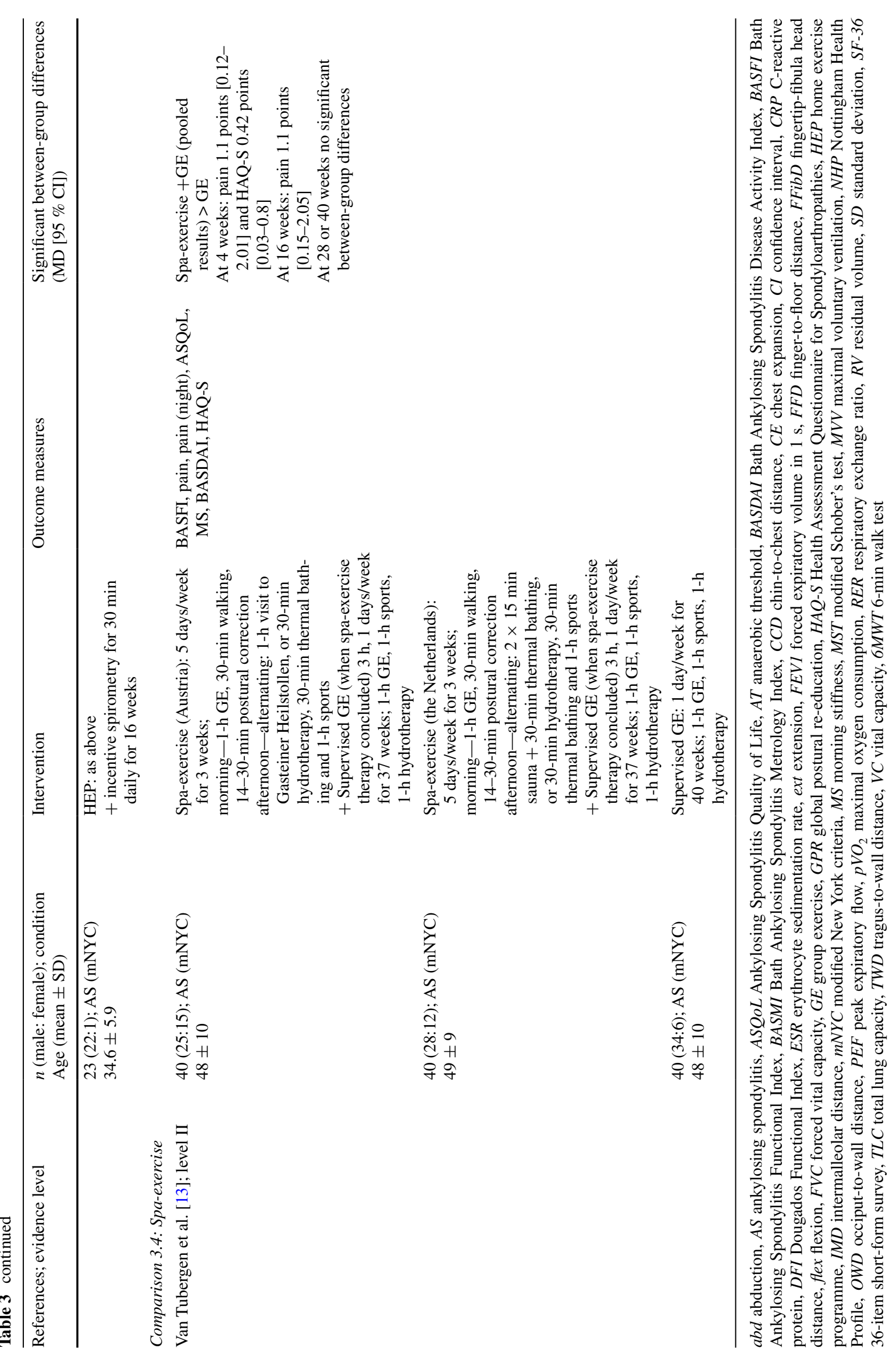


Fig. 2 Risk of bias of included studies

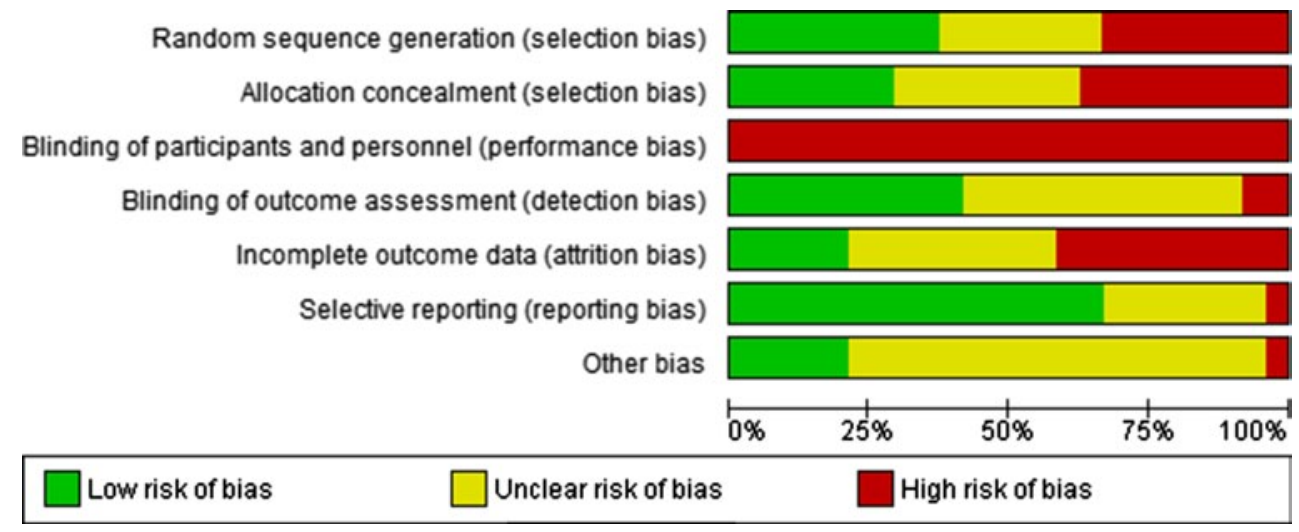

results from both exercise groups were pooled for comparison with controls [12].

\section{Function, QoL, disease activity, pain and stiffness}

Physical function was measured using the Bath Ankylosing Spondylitis Functional Index (BASFI) in six of the seven studies. Four of these are presented in Fig. 3; only the study by Durmus et al. [35] significantly favoured exercise. The other two studies did not report mean and standard deviations and are not included in the figure; their findings favoured exercise over controls [28, 30]. Benefits were maintained at 6-month follow-up [30].

The results of four studies measuring disease activity on the Bath Ankylosing Spondylitis Disease Activity Index (BASDAI) are presented in Fig. 3; the study by Durmus et al. [35] significantly favoured the exercise group. Masiero et al. [30] also found BASDAI scores to be significantly improved immediately after GE, and at 6-month follow-up, compared to controls. Compared to controls, spinal mobility scores on the Bath Ankylosing Spondylitis Metrology Index (BASMI) were significantly lower following a rehabilitation programme [30], but not significantly different after a Pilates intervention [34].

In comparison with controls, QoL was significantly improved following a HEP [35], but not following a Pilates-based intervention [34]. Pain and stiffness scores were significantly lower following exercise interventions and at 6-month follow-up [28, 30].

\section{Health-related fitness outcomes}

Following a 3-month multimodal GE intervention, a significant improvement was observed in physical work capacity on a bicycle ergometer and predicted vital capacity [36]. Durmus et al. [12] reported a significant increase in distance walked in a 6-min walk test (6MWT) following exercise compared to controls; no significant between-group

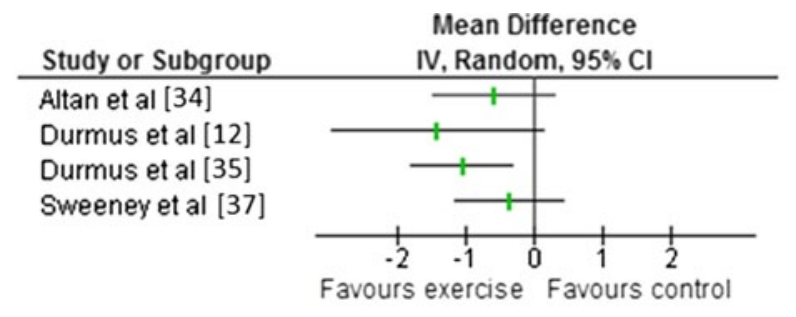

a Exercise compared to controls in BASFI

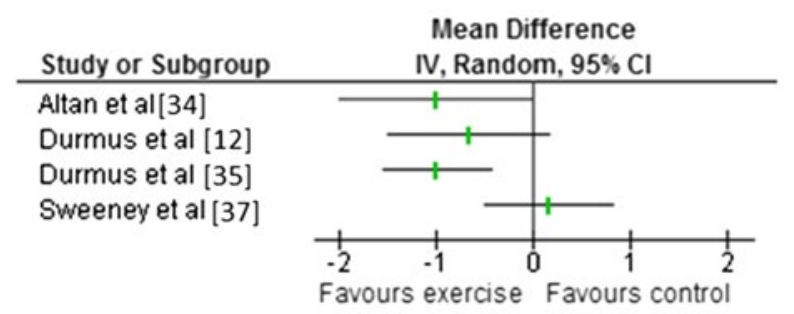

b Exercise compared to controls in BASDAI

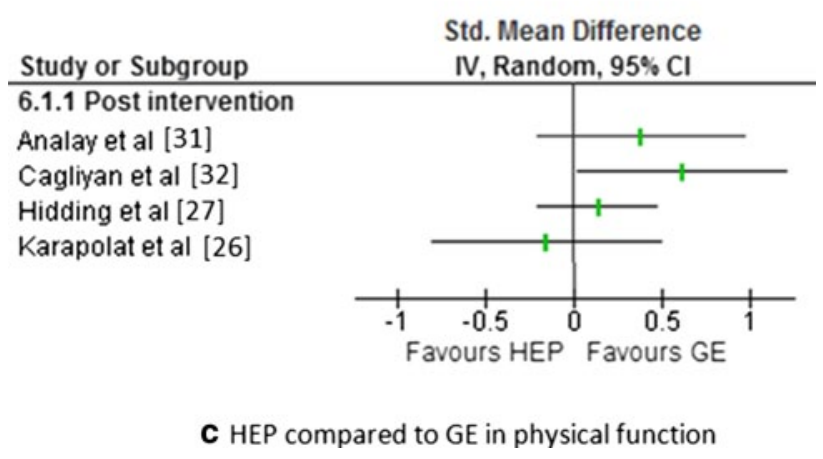

Fig. 3 Forest plot of between-group comparisons. Std standardised

differences were observed in pulmonary function tests (PFTs).

The majority of individual mobility tests were significantly improved after exercise compared to controls [28, 36]. Inclinometer and pocket goniometry assessment found significant improvements favouring exercise groups in 
cervical, shoulder and knee range of motion (ROM), but results for thoraco-lumbar mobility were conflicting [28, $30,36]$. Chest expansion (CE) was significantly greater in a HEP group [12], a group undergoing a multimodal intervention [36], a rehabilitation group [30], but not in a Pilates group [34], compared to controls.

Therapeutic exercise is effective for improving physical function, disease activity and CE compared to controls, and the level of evidence is moderate coming from six, five and four studies, respectively. Exercise programmes are effective at improving joint mobility, cardiorespiratory function, pain and stiffness, and the level of evidence is low. There is conflicting evidence as to the effect of therapeutic exercise on QoL.

Comparison 2.1: unsupervised HEP compared to supervised GE

Three studies compared the effectiveness of unsupervised HEP with supervised GE [26, 31, 32]. One study evaluated the effects of adding weekly GE to a HEP [27]. One threearmed study compared regular supervised GE, infrequent supervised individual exercise supplemented with a HEP and unsupervised HEP [20].

\section{Function, QoL, disease activity, pain and stiffness}

Physical function was assessed in four studies using different outcome measures (Fig. 3). Cagliyan et al. [32] found a significant difference favouring the GE, which was maintained at 3-month follow-up. They also found that QoL significantly favoured GE immediately after a 3-month intervention; only physical role difficulty subscore remained significantly superior at follow-up. Karapolat et al. [26] found GE to be equivalent to HEP in QoL, except in the sleep subscore in which GE was significantly superior.

Disease activity (BASDAI) was significantly lower following GE compared to HEP in the study by Cagliyan et al. [32]; however, Karapolat et al. [26] found no significant between-group differences. The effects of GE and HEP on resting pain, pain during activities and duration of morning stiffness were comparable [27, 31, 32].

\section{Health-related fitness outcomes}

No intergroup differences in spinal mobility were found across a variety of measures [20, 26, 31]. Cagliyan et al. [32] found that $\mathrm{CE}$ and finger-to-floor distance favoured HEP over $\mathrm{GE}$, whereas intermalleolar distance was superior following GE; no differences were found at follow-up. Thoraco-lumbar flexion/extension was significantly superior following the addition of weekly GE to a HEP compared to HEP alone. Cervical rotation and CE were similar across groups [20, 27].
Hidding et al. [27] measured aerobic power with a maximal, incremental exercise test on a cycle ergometer. Maximum workload was significantly higher following GE compared to HEP. Analay et al. [31] compared $\mathrm{VO}_{2 \mathrm{MAX}}$ values obtained by the Astrand test and found no significant between-group differences after exercise interventions or at follow-up.

Group exercise is more beneficial than HEP in improving QoL, and the level of evidence is moderate coming from two studies. There is no difference between supervised GE and HEP in physical function, pain and stiffness, and the level of evidence is moderate. There is no difference between GE and HEP for most spinal mobility, but the level of evidence is low. The findings of studies assessing disease activity and cardiorespiratory fitness are conflicting.

Comparison 2.2: global postural re-education

\section{Function, QoL, disease activity, pain and stiffness}

Three studies compared group GPR to GE [21, 22, 33], and one study compared individual GPR with GE [23]. This latter study favoured individual GPR over GE in physical function, physical aspects of QoL, pain scores and morning stiffness duration, but the level of evidence is very low coming from this single level III study. There was no significant difference between-group GPR and GE programmes in physical function and disease activity outcomes, and the level of evidence is moderate from one level II study [33]. There was no difference in the effectiveness of group GPR and GE programmes in QoL, but the level of evidence was very low coming from one level III study [21].

\section{Health-related fitness outcomes}

There is very low evidence coming from one level III study [23] that individual GPR is superior to conventional GE in improving spinal mobility and CE. No significant betweengroup differences in spinal mobility or PFTs were reported; the level of evidence for no difference is moderate and very low, respectively $[22,33]$.

\section{Comparison 2.3: aerobic exercise}

Karapolat et al. [38] investigated the effects of adding aerobic exercise to a stretching and mobility HEP. The addition of swimming to a HEP significantly increased walking distance on 6MWT test compared to HEP alone, but no significant between-group differences were observed in cardiorespiratory variables. Niedermann et al. [29] found that the addition of aerobic training to a flexibility programme increased cardiorespiratory fitness measured with a submaximal bicycle test, but did not result in a significant 
difference in cardiovascular risk factors (cholesterol and triglycerides). There is no significant between-group differences in disease activity, quality of life or spinal mobility, and the level of evidence is moderate coming from two level II studies.

\section{Comparison 2.4: multimodal exercise programmes}

Roşu et al. [39] compared two multimodal HEP. A group performing a multimodal HEP of breathing, postural and stretching exercises (based on the Pilates, Heckscher and McKenzie methods) had significantly improved disease activity, physical function, spinal mobility and vital capacity compared to an exercise programme that combined step-aerobics and stretching. The risk of bias within this study is high, and the quality of evidence is low.

Comparison 3: therapeutic exercise compared to other modalities

\section{Inpatient rehabilitation}

Figen et al. [19] compared a 3-week HEP with 3 weeks of inpatient rehabilitation. Will et al. [18] compared high-frequency inpatient exercise with supervised outpatient exercise, both supplemented by HEP. Comprehensive inpatient rehabilitation did not significantly change physical function, disease activity or spinal mobility compared to outpatient exercise; the level of evidence of no difference between HEP and inpatient rehabilitation is moderate coming from two studies.

\section{Balneotherapy}

Altan et al. [24] compared the effect of balneotherapy and HEP to HEP alone. Disease activity and QoL were significantly improved in the balneotherapy and HEP groups immediately after intervention, but equivalent at 24-week follow-up. No significant group differences were found in physical function, pain, morning stiffness duration or spinal mobility. The level of evidence is moderate coming from one level II study.

\section{Incentive spirometry}

The addition of incentive spirometry exercises to a HEP did not significantly improve physical function (BASFI or 6MWT), disease activity, PFTs or spinal mobility compared to HEP alone [25]; the level of evidence of no difference is moderate coming from one level II study.

\section{Spa-exercise}

Van Tubergen et al. [13] compared the effects of two different, 3-week spa-exercise protocols (followed by 37 weeks of GE) with supervised GE. Data for the spa-exercise groups were pooled and compared to the supervised GE group. Pain and HAQ-S scores favoured the spa-exercise groups after the initial 3-week treatment at the spa centres. Pain remained significantly improved at 16 weeks, but this difference was no longer present 28 and 40 weeks into the intervention. The level of evidence from this single level II study is moderate.

\section{Discussion}

This review found evidence that therapeutic exercise has greater benefits than no intervention in improving physical function, disease activity, pain, stiffness, joint mobility and cardiovascular performance in adults with AS; evidence from trials examining QoL is conflicting. Exercise conducted under supervision has benefits over unsupervised HEP for QoL, but there is evidence of no difference, and conflicting evidence, across other outcomes. Spa-exercise and balneotherapy programmes have short-term benefits in QoL outcomes compared to GE; spa-exercise is also superior in pain relief, while balneotherapy further improves disease activity. Results from inpatient rehabilitation protocols were comparable to outpatient exercise protocols. These findings are in keeping with previous reviews $[8,40]$.

In comparisons of different exercise regimes, the addition of aerobic exercise to conventional stretching and mobility HEP results in superior functional fitness. Supplementing HEP with daily incentive spirometry does not yield additional benefits. Studies investigating different flexibility programmes have a high risk of bias; the GPR method delivered on an individual basis and a multimodal stretching and mobility programme appear superior to conventional exercise programmes.

The heterogeneity of exercise protocols and outcome measures employed preclude firm conclusions being drawn on the most effective exercise prescription. Vague descriptions of exercise protocols coupled with suboptimal dosage of exercise prescription, below that recommended to elicit physiological changes, add to the difficulty in assessing the impact of therapeutic exercise on SpA [41]. Furthermore, under-reporting of adherence to programmes was a feature of the included studies, making determining the efficacy of interventions problematic.

The outcome measures reported are principally selfreport in nature, with few studies assessing physiological measures. The investigation of the effects of exercise interventions on health-related fitness has centred on flexibility and cardiorespiratory domains; the effect of exercise on muscular strength and endurance, and body composition has not been investigated. Despite the increase in cardiovascular morbidity and mortality among SpA populations, 
the potential benefits of therapeutic exercise programmes on cardiovascular risk factor are yet to be adequately investigated in SpA [9].

Most benefits observed immediately post-intervention were not maintained at follow-up. The chronic nature of $\mathrm{SpA}$ requires ongoing, regular exercise; however, the optimal frequency necessary to maintain benefits is unknown. In the last two decades, the emergence of biologics has dramatically changed pharmacological approaches to the management of SpA. With improved management of inflammatory symptoms, there has been decreased compliance with exercise [42]. Regular involvement in exercise also declines with increased disease duration [43]; long-term compliance with exercise, particularly in people with lower disability levels, presents a clinical challenge.

\section{Study limitations and future research}

Non-randomised controlled trials were included in this review to increase the scope of the review, but simultaneously increased the risk of bias. It is a further constraint that data extraction was performed by one reviewer. In practice, therapeutic exercise is frequently prescribed as part of a multimodal treatment plan [30, 44]; this review excluded studies examining exercise therapy in combination with other modalities as the relative effect of exercise therapy would be unknown. Combining exercise prescription with other modalities may yield different outcomes.

Participants in the included studies were almost exclusively diagnosed with AS; extrapolating findings to other SpA subtypes should be undertaken cautiously. Future studies should account for the evolving classification of $\mathrm{SpA}$ (e.g. predominantly axial $\mathrm{SpA}$ or predominantly peripheral $\mathrm{SpA}$ ) and explore the effects of exercise on other SpA subtypes. Furthermore, comprehensive reporting of exercise protocols and participant adherence rates in studies is essential to understanding the effectiveness of exercise therapy. Targeted exercise prescription should meet the dosage recommended to achieve physiological changes. Methodological quality among studies in this review was mixed. Random sequence generation, adequate allocation concealment and blinding of outcome assessment in future RCTs would go some way towards addressing methodological shortcomings.

\section{Conclusions}

Current evidence shows therapeutic exercise to be beneficial for adults with AS, although the effects on other SpA subtypes are unknown. Expanding traditional programmes of flexibility exercises to include aerobic components may improve clinical outcomes, although the most effective exercise protocol remains unclear.
Conflict of interest The authors declare that they have no conflict of interest.

\section{References}

1. Zochling J, Brandt J, Braun J (2005) The current concept of spondyloarthritis with special emphasis on undifferentiated spondyloarthritis. Rheumatology (Oxford, England) 44(12):1483-1491

2. Singh JA, Strand V (2009) Spondyloarthritis is associated with poor function and physical health-related quality of life. J Rheumatol 36(5):1012-1020. doi:10.3899/jrheum.081015

3. Heikkila S, Viitanen JV, Kautiainen H, Kauppi M (2002) Functional long-term changes in patients with spondyloarthropathy. Clin Rheumatol 21(2):119-122

4. Rudwaleit M, van der Heijde D, Landewe R, Listing J, Akkoc N, Brandt J, Braun J, Chou CT, Collantes-Estevez E, Dougados M, Huang F, Gu J, Khan MA, Kirazli Y, Maksymowych WP, Mielants H, Sorensen IJ, Ozgocmen S, Roussou E, Valle-Onate R, Weber U, Wei J, Sieper J (2009) The development of Assessment of SpondyloArthritis international Society classification criteria for axial spondyloarthritis (part II): validation and final selection. Ann Rheum Dis 68(6):777-783. doi:10.1136/ard.2009.108233

5. Rudwaleit M, van der Heijde D, Landewe R, Akkoc N, Brandt J, Chou CT, Dougados M, Huang F, Gu J, Kirazli Y, Van den Bosch F, Olivieri I, Roussou E, Scarpato S, Sorensen IJ, Valle-Onate R, Weber U, Wei J, Sieper J (2011) The Assessment of SpondyloArthritis International Society classification criteria for peripheral spondyloarthritis and for spondyloarthritis in general. Ann Rheum Dis 70(1):25-31. doi:10.1136/ard.2010.133645

6. Braun J, van den Berg R, Baraliakos X, Boehm H, Burgos-Vargas R, Collantes-Estevez E, Dagfinrud H, Dijkmans B, Dougados M, Emery P, Geher P, Hammoudeh M, Inman RD, Jongkees M, Khan MA, Kiltz U, Kvien T, Leirisalo-Repo M, Maksymowych WP, Olivieri I, Pavelka K, Sieper J, Stanislawska-Biernat E, Wendling D, Ozgocmen S, van Drogen C, van Royen B, van der Heijde D (2011) 2010 update of the ASAS/EULAR recommendations for the management of ankylosing spondylitis. Ann Rheum Dis 70(6):896-904. doi:10.1136/ard.2011.151027

7. Ritchlin CT, Kavanaugh A, Gladman DD, Mease PJ, Helliwell P, Boehncke WH, de Vlam K, Fiorentino D, Fitzgerald O, Gottlieb AB, McHugh NJ, Nash P, Qureshi AA, Soriano ER, Taylor WJ (2009) Treatment recommendations for psoriatic arthritis. Ann Rheum Dis 68(9):1387-1394. doi:10.1136/ard.2008.094946

8. Dagfinrud H, Kvien TK, Hagen KB (2008) Physiotherapy interventions for ankylosing spondylitis. Cochrane Database Syst Rev (1), Art No CD002822. doi:10.1002/14651858.CD002822.pub3

9. Papagoras C, Voulgari PV, Drosos AA (2013) Atherosclerosis and cardiovascular disease in the spondyloarthritides, particularly ankylosing spondylitis and psoriatic arthritis. Clin Exp Rheumatol 31(4):612-620

10. Han C, Robinson DW Jr, Hackett MV, Paramore LC, Fraeman $\mathrm{KH}$, Bala MV (2006) Cardiovascular disease and risk factors in patients with rheumatoid arthritis, psoriatic arthritis, and ankylosing spondylitis. J Rheumatol 33(11):2167-2172

11. Higgins JPT, Green S (eds) (2011) Cochrane handbook for systematic reviews of interventions, Version 5.1.0 [updated March 2011]. The Cochrane Collaboration. Available from http://www.cochrane-handbook.org

12. Durmus D, Alayli G, Uzun O, Tander B, Canturk F, Bek Y, Erkan L (2009) Effects of two exercise interventions on pulmonary functions in the patients with ankylosing spondylitis. Joint Bone Spine 76(2):150-155. doi:10.1016/j.jbspin.2008.06.013

13. van Tubergen A, Landewe R, van der Heijde D, Hidding A, Wolter N, Asscher M, Falkenbach A, Genth E, The HG, van der 
Linden S (2001) Combined spa-exercise therapy is effective in patients with ankylosing spondylitis: a randomized controlled trial. Arthritis Rheum 45(5):430-438

14. Macedo LG, Elkins MR, Maher CG, Moseley AM, Herbert RD, Sherrington C (2010) There was evidence of convergent and construct validity of Physiotherapy Evidence Database quality scale for physiotherapy trials. J Clin Epidemiol 63(8):920-925. doi:10.1016/j.jclinepi.2009.10.005

15. Maher CG, Sherrington C, Herbert RD, Moseley AM, Elkins M (2003) Reliability of the PEDro scale for rating quality of randomized controlled trials. Phys Ther 83(8):713-721

16. Oxford Centre for Evidence Based Medicine: Levels of Evidence Working Group (2011) The Oxford Levels of Evidence. http://www.cebm.net/index.aspx?o=5653. Accessed 1 Aug 2013

17. Balshem H, Helfand M, Schunemann HJ, Oxman AD, Kunz R, Brozek J, Vist GE, Falck-Ytter Y, Meerpohl J, Norris S, Guyatt GH (2011) GRADE guidelines: 3. Rating the quality of evidence. J Clin Epidemiol 64(4):401-406. doi:10.1016/j.jclinepi.2010.07.015

18. Will RK, Suryana BPP, Lim A, Walsh E, Buchanan J (2003) Intensive exercise in patients with spondyloarthropathies-a randomized controlled study. Paper presented at the European League Against Rheumatism (EULAR) Annual Congress, Lisbon

19. Figen A, Gecene M, Gunduz R, Borman P, Yorgancioglu R (2011) Long-term effects of comprehensive inpatient rehabilitation on function and disease activity in patients with chronic rheumatoid arthritis and ankylosing spondylitis. Turk J Rheumatol 26(2):135-144

20. Ramos-Solchaga M, Ossorio Castellanos C, Garcia Soro JM (1998) Influencia de la terapia fisica mediante ejercicios en la evolucion a largo plazo de la espondilitis anquilosante. Rehabilitación (Madr) 32(5):316-323

21. Rivera-Navarro J, Fernandez-De-Las-Penas C, Alonso-Blanco C, Miangolarra-Page JC (2005) Repercusiones en la calidad de vida en pacientes con espondilitis anquilosante mediante tratamiento fisioterápico. Fisioterapia 27(3):138-145

22. Alonso-Blanco C, Rodriguez-López ESR, Fernández-de-lasPenas C (2009) Cambios espirometricos tras la aplicacion de un programa de cinesiterapia en la espondilitis anquilosante: estudio piloto. Fisioterapia 31(3):87-93

23. Silva EM, Andrade SC, Vilar MJ (2012) Evaluation of the effects of Global Postural Reeducation in patients with ankylosing spondylitis. Rheumatol Int 32(7):2155-2163

24. Altan L, Bingol U, Aslan M, Yurtkuran M (2006) The effect of balneotherapy on patients with ankylosing spondylitis. Scand J Rheumatol 35(4):283-289

25. So MW, Heo HM, Koo BS, Kim YG, Lee CK, Yoo B (2012) Efficacy of incentive spirometer exercise on pulmonary functions of patients with ankylosing spondylitis stabilized by tumor necrosis factor inhibitor therapy. J Rheumatol 39(9):1854-1858

26. Karapolat H, Akkoc Y, Sari I, Eyigor S, Akar S, Kirazli Y, Akkoc $\mathrm{N}$ (2008) Comparison of group-based exercise versus home-based exercise in patients with ankylosing spondylitis: effects on Bath Ankylosing Spondylitis Indices, quality of life and depression. Clin Rheumatol 27(6):695-700. doi:10.1007/s10067-007-0765-0

27. Hidding A, van der Linden S, Boers M, Gielen X, de Witte L, Kester A, Dijkmans B, Moolenburgh D (1993) Is group physical therapy superior to individualized therapy in ankylosing spondylitis? Arthritis Care Res (Hoboken) 6(3):117-125

28. Lim HJ, Moon YI, Lee MS (2005) Effects of home-based daily exercise therapy on joint mobility, daily activity, pain, and depression in patients with ankylosing spondylitis. Rheumatol Int 25(3):225-229

29. Niedermann K, Sidelnikov E, Muggli C, Dagfinrud H, Hermann M, Tamborrini G, Ciurea A, Bischoff-Ferrari H (2013)
Cardiovascular training improves fitness in patients with ankylosing spondylitis. Arthritis Care Res (Hoboken). doi:10.1002/ acr. 22062

30. Masiero S, Bonaldo L, Pigatto M, lo Nigro A, Ramonda R, Punzi L (2011) Rehabilitation treatment in patients with ankylosing spondylitis stabilized with tumor necrosis factor inhibitor therapy. A randomized controlled trial. J Rheumatol 38(7):13351342. doi:10.3899/jrheum. 100987

31. Analay Y, Ozcan E, Karan A, Diracoglu D, Aydin R (2003) The effectiveness of intensive group exercise on patients with ankylosing spondylitis [with consumer summary]. Clin Rehabil 17(6):631-636

32. Cagliyan A, Kotevoglu N, Onal T, Tekkus B, Kuran B (2007) Does group exercise program add anything more to patients with ankylosing spondylitis? J Back Musculoskelet Rehabil 20(2-3):79-85

33. Fernandez-de-las-Penas C, Alonso-Blanco C, Morales-Cabezas M, Miangolarra-Page JC (2005) Two exercise interventions for the management of patients with ankylosing spondylitis: a randomized controlled trial. Am J Phys Med Rehabil 84(6):407-419

34. Altan L, Korkmaz N, Dizdar M, Yurtkuran M (2012) Effect of Pilates training on people with ankylosing spondylitis. Rheumatol Int 32(7):2093-2099

35. Durmus D, Alayli G, Cil E, Canturk F (2009) Effects of a homebased exercise program on quality of life, fatigue, and depression in patients with ankylosing spondylitis. Rheumatol Int 29(6):673-677

36. Ince G, Sarpel T, Durgun B, Erdogan S (2006) Effects of a multimodal exercise program for people with ankylosing spondylitis. Phys Ther 86(7):924-935

37. Sweeney S, Taylor G, Calin A (2002) The effect of a home based exercise intervention package on outcome in ankylosing spondylitis: a randomized controlled trial. J Rheumatol 29(4):763-766

38. Karapolat H, Eyigor S, Zoghi M, Akkoc Y, Kirazli Y, Keser G (2009) Are swimming or aerobic exercise better than conventional exercise in ankylosing spondylitis patients? A randomized controlled study. Eur J Phys Rehabil Med 45(4):449-457

39. Rosu MO, Topa I, Chirieac R, Ancuta C (2013) Effects of Pilates, McKenzie and Heckscher training on disease activity, spinal motility and pulmonary function in patients with ankylosing spondylitis: a randomized controlled trial. Rheumatol Int. doi:10.1007/s00296-013-2869-y

40. Ribeiro F, Leite M, Silva F, Sousa O (2007) Physical exercise in the treatment of Ankylosing Spondylitis: a systematic review. Acta Reumatol Port 32(2):129-137

41. Dagfinrud H, Halvorsen S, Vollestad NK, Niedermann K, Kvien TK, Hagen KB (2011) Exercise programs in trials for patients with ankylosing spondylitis: do they really have the potential for effectiveness? Arthritis Care Res (Hoboken) 63(4):597-603. doi:10.1002/acr.20415

42. Falkenbach A (2003) Disability motivates patients with ankylosing spondylitis for more frequent physical exercise. Arch Phys Med Rehabil 84(3):382-383

43. Passalent LA, Soever LJ, O'Shea FD, Inman RD (2010) Exercise in ankylosing spondylitis: discrepancies between recommendations and reality. J Rheumatol 37(4):835-841. doi:10.3899/jrh eum.090655

44. Rodriguez-Lozano C, Juanola X, Cruz-Martinez J, Pena-Arrebola A, Mulero J, Gratacos J, Collantes E (2013) Outcome of an education and home-based exercise programme for patients with ankylosing spondylitis: a nationwide randomized study. Clin Exp Rheumatol 31(5):739-748 